\title{
Differences in Medicare Beneficiary Risk Scores by Physician's International Medical Graduate Status
}

\author{
McKinley Glover IV, MD, MHS ${ }^{1,2,3}$, Nathaniel D. Mercaldo, $P h D^{1,2}$, \\ Daniel M. Blumenthal, MD, MBA ${ }^{2,4,5}$, Timothy G. Ferris, MD, MPH 2,3,6, and Jason H. Wasfy, MD, \\ MPhil,3,4
}

\begin{abstract}
'Department of Radiology, Massachusetts General Hospital, Boston, MA, USA; ${ }^{2}$ Harvard Medical School, Boston, MA, USA; ${ }^{3}$ Massachusetts General Physicians Organization, Boston, MA, USA; ${ }^{4}$ Cardiology Division, Massachusetts General Hospital, Boston, MA, USA; 5 Devoted Health, Waltham, MA, USA; ${ }^{6}$ Department of Internal Medicine, Massachusetts General Hospital, Boston, MA, USA.
\end{abstract}

J Gen Intern Med 34(7):1110-2

DOI: $10.1007 /$ s11606-019-04830-0

(C) Society of General Internal Medicine 2019

\section{INTRODUCTION}

International medical graduates (IMGs) comprise nearly one-fourth of US practicing physicians and are more likely to practice primary care and in underserved regions. ${ }^{1,2}$ Despite evidence on the quality of care provided by IMGs, limited data exists on whether populations served by IMGs have different medical complexity than those served by domestic graduates (DGs). ${ }^{3}$ Understanding differences in populations served by IMGs and DGs is important when assessing the potential impact of federal immigration policies on IMGs and the patients they serve. Therefore, we examined the relationship between physician IMG status and Medicare beneficiary medical complexity, among internal medicine (IM) physicians.

\section{METHODS}

We performed a cross-sectional analysis of US physicians within Centers for Medicare \& Medicaid Services (CMS) Physician Compare in 2015, which contains demographic and practice information for all healthcare providers participating in Medicare. Data included gender, specialty, medical school graduation year, practice location, and claims-based hospital affiliations. Physicians reported as IM were included, excluding those also reporting IM subspecialties. Using claims-based affiliations, physicians were stratified by teaching status (major, minor, none). Medicare utilization data was obtained from the 2015 Medicare Provider Utilization and Payment Data File (PUF). Characterization as a DG or IMG was based on each physician's medical school, obtained from Doximity data.

The main outcome variable was average CMS Hierarchical Condition Category (HCC) risk score of all unique Medicare beneficiaries for each physician, as reported in the PUF. The CMS-HCC model, using demographic

Published online February 8, 2019 information and diagnoses, generates risk scores for each beneficiary that summarize expected cost of care relative to an average beneficiary (risk score $=1.0$ ). A risk score of 1.5 would predict expenditures to be $50 \%$ higher than an average beneficiary. Average CMS-HCC risk score for each physician was used for several reasons: public availability, importance in value-based payment models, and use as a proxy for health status. ${ }^{4}$

A multivariable quantile regression model was constructed, estimating the association between average beneficiary HCC risk score and IMG status as well as additional physician and practice characteristics. Based on positive skewness of the outcome variable, linear regression modeling assumptions regarding normally distributed residuals were violated. Thus, quantile regression was employed as a more robust approach to estimate average HCC risk score.

Multiple imputation was employed to account for unknown IMG status and was included in the final regression model. Sensitivity analyses were performed in which unknown IMG status was imputed as DG and then as IMG. Statistical analyses were performed using $\mathrm{R}$ 3.4.3 (mice, quantreg libraries).

\section{RESULTS}

Among 74,999 IM physicians, $51.8 \%$ were DGs, $29.7 \%$ were IMGs, and $18.5 \%$ had unknown IMG status (Table 1). The overall median of average beneficiary HCC risk score was 1.73 (IQR 1.20, 2.39). The unadjusted median of average beneficiary HCC risk score was 1.45 (IQR 1.11, 2.23) among DGs and 1.94 (IQR 1.32, 2.46) among IMGs. When adjusting for covariates and accounting for unknown IMG status, the median IMG average beneficiary HCC risk scores were 0.30 (95\% CI 0.29-0.31) units higher than DGs (Table 2). Sensitivity analyses assessing the impact of unknown IMG status resulted in attenuated estimates of changes in median IMG average beneficiary HCC risk scores (i.e., unknown $=$ DG $0.26(95 \%$ CI $0.25-0.27)$ and unknown $=$ IMG 0.27 (95\% CI 0.26-0.29). 
Table 1 Characteristics of Internal Medicine Physicians in Study Sample by International Medical Graduate Status $(N=\mathbf{7 4 , 9 9 9 )}$

\begin{tabular}{|c|c|c|c|}
\hline Characteristics & $\begin{array}{l}\text { Domestic } \\
\text { medical } \\
\text { graduate } \\
N=\mathbf{3 8 , 8 8 6}\end{array}$ & $\begin{array}{l}\text { International } \\
\text { medical } \\
\text { graduate } \\
N=22,274\end{array}$ & $\begin{array}{c}\text { Unknown } \\
N=\mathbf{1 3 , 8 3 9}\end{array}$ \\
\hline \multicolumn{4}{|l|}{ Gender } \\
\hline Male & $24,886(64)$ & $14,401(65)$ & $8059(58)$ \\
\hline Female & $14,000(36)$ & $7873(35)$ & $5780(42)$ \\
\hline \multicolumn{4}{|c|}{ Years since residency completion } \\
\hline $0-10$ & $10,546(27)$ & 4277 (19) & $7945(57)$ \\
\hline $11-20$ & $12,148(31)$ & $7286(33)$ & $3061(22)$ \\
\hline $21-30$ & $9261(24)$ & $6633(30)$ & $1790(13)$ \\
\hline $31-40$ & $5827(15)$ & 3113 (14) & $809(6)$ \\
\hline $40+$ & $1104(3)$ & $965(4)$ & $234(2)$ \\
\hline \multicolumn{4}{|l|}{ Teaching affiliation } \\
\hline None & $10,717(28)$ & $6932(31)$ & $3797(27)$ \\
\hline Minor & $14,320(37)$ & $8635(39)$ & $5310(38)$ \\
\hline Major & $13,849(36)$ & $6707(30)$ & $4732(34)$ \\
\hline \multicolumn{4}{|l|}{ Rurality } \\
\hline Urban & $34,867(90)$ & $20,229(91)$ & $12,571(91)$ \\
\hline Rural & $4019(10)$ & $2045(9)$ & $1268(9)$ \\
\hline \multicolumn{4}{|l|}{ Region } \\
\hline Midwest & $8164(21)$ & $4853(22)$ & $3120(23)$ \\
\hline Northeast & $8935(23)$ & $6114(27)$ & $3973(29)$ \\
\hline South & $13,096(34)$ & $7668(34)$ & $4285(31)$ \\
\hline West & $8691(22)$ & 3639 (16) & $2461(18)$ \\
\hline \multicolumn{4}{|c|}{ Total Medicare services provided ${ }^{\mathrm{a}}$} \\
\hline $0-500$ & $10,342(27)$ & $4846(22)$ & $4447(32)$ \\
\hline $501-1000$ & $8210(21)$ & $4580(21)$ & $3405(25)$ \\
\hline $1001-2500$ & $11,028(28)$ & $7839(35)$ & $4209(30)$ \\
\hline $2501+$ & $9306(24)$ & $5009(22)$ & $1778(13)$ \\
\hline \multicolumn{4}{|c|}{ Total unique Medicare beneficiaries } \\
\hline $0-100$ & $6084(16)$ & 3050 (14) & $2428(18)$ \\
\hline $101-250$ & $10,898(28)$ & $5680(26)$ & $3921(28)$ \\
\hline $251-500$ & $13,850(36)$ & $8322(37)$ & $4988(36)$ \\
\hline $501+$ & $8054(21)$ & $5222(23)$ & $2502(18)$ \\
\hline \multicolumn{4}{|c|}{ Total Medicare allowed amount $(\$)^{\mathrm{b}}$} \\
\hline $0-50,000$ & $12,080(31)$ & $5516(25)$ & $4750(34)$ \\
\hline $50,001-100,000$ & $9869(25)$ & $5104(24)$ & $3671(27)$ \\
\hline 100,000 & $6697(17)$ & $4200(19)$ & $2492(18)$ \\
\hline \multicolumn{4}{|l|}{150,000} \\
\hline $150,001+$ & $10,240(26)$ & 7454 (33) & $2926(21)$ \\
\hline
\end{tabular}

${ }^{a}$ Total services represents total number of CPT codes billed by each

${ }^{b}$ Total allowed amount equals sum of the following: Medicare payment, beneficiary payments (coinsurance and/or deductible), and any amount a third party is responsible for provider in 2015

Table 2 Multivariable Quantile Regression Model: Average Beneficiary CMS HCC Risk Score of Internal Medicine Physicians in $2015(N=74,999)$

\begin{tabular}{|c|c|c|}
\hline $\begin{array}{l}\text { Physician } \\
\text { characteristics }\end{array}$ & $\begin{array}{l}\text { Estimate of average } \\
\text { HCC risk score of } \\
\text { Medicare } \\
\text { beneficiaries }\end{array}$ & $95 \% \mathrm{CI}$ \\
\hline Intercept & 2.02 & $1.99,2.04$ \\
\hline \multicolumn{3}{|l|}{ Medical school } \\
\hline $\begin{array}{l}\text { International medical } \\
\text { graduate }\end{array}$ & 0.30 & $0.29,0.31$ \\
\hline \multicolumn{3}{|l|}{ Gender } \\
\hline Female & -0.16 & $-0.17,-0.15$ \\
\hline \multicolumn{3}{|c|}{ Years since residency completion } \\
\hline $0-10$ & Reference & \\
\hline $11-20$ & -0.36 & $-0.38,-0.35$ \\
\hline $21-30$ & -0.61 & $-0.62,-0.59$ \\
\hline $31-40$ & -0.67 & $-0.69,-0.65$ \\
\hline \multirow{2}{*}{\multicolumn{3}{|c|}{ Teaching affiliation }} \\
\hline & & \\
\hline None & Reference & \\
\hline Minor & 0.13 & $0.11,0.14$ \\
\hline Major & 0.15 & $0.13,0.16$ \\
\hline \multicolumn{3}{|l|}{ Rurality } \\
\hline Urban/suburban & Reference & \\
\hline Rural & -0.06 & $-0.08,-0.04$ \\
\hline \multicolumn{3}{|l|}{ Region } \\
\hline Midwest & Reference & \\
\hline Northeast & -0.06 & $-0.08,-0.04$ \\
\hline South & 0.06 & $0.05,0.08$ \\
\hline West & 0.02 & $0.00,0.04$ \\
\hline \multicolumn{3}{|c|}{ Total Medicare services provided } \\
\hline $0-500$ & Reference & \\
\hline $501-1000$ & -0.38 & $-0.41,-0.36$ \\
\hline $1001-2500$ & -0.80 & $-0.82,-0.77$ \\
\hline $2501+$ & -1.22 & $-1.26,-1.19$ \\
\hline \multicolumn{3}{|c|}{ Total unique Medicare beneficiaries } \\
\hline $0-100$ & Reference & \\
\hline $101-250$ & 0.09 & $0.07,0.11$ \\
\hline $251-500$ & 0.23 & $0.21,0.26$ \\
\hline $501+$ & 0.34 & $0.31,0.37$ \\
\hline \multicolumn{3}{|c|}{ Total Medicare allowed amount } \\
\hline $0-50,000$ & Reference & \\
\hline $50,00-100,000$ & 0.36 & $0.33,0.38$ \\
\hline $100,000-150,000$ & 0.57 & $0.54,0.60$ \\
\hline $150,001+$ & 0.67 & $0.69,0.71$ \\
\hline
\end{tabular}

or their socioeconomic status. Despite multiple imputation and sensitivity analyses, unknown IMG status remains a limitation. Potential differences in coding and documentation practices may also confound the findings, warranting additional study.

IMGs practicing IM had significantly higher average Medicare beneficiary CMS HCC risk scores than DGs, after adjustment for several physician and practice characteristics. Implementation of federal immigration policies that affect current and prospective IMGs may adversely impact access to care for Medicare beneficiaries with poorer health. IMGs may already face overt and subtle workplace bias and discrimination and professional limitations. ${ }^{5}$ Additionally, a milieu that reduces prospective IMGs from seeking to practice in the USA could exacerbate the physician shortage, particularly in primary care. ${ }^{6}$

Study limitations include using average CMS-HCC risk scores, which may not fully reflect patient medical complexity. Further, average CMS-HCC risk score does not account for volume or intensity of care provided to individual beneficiaries
Corresponding Author: McKinley Glover, IV, MD, MHS; Massachusetts General Physicians Organization, Boston, MA, USA (e-mail: mckinley.glover@mgh.harvard.edu).

Funding Source(s) Statement No direct sources of funding were obtained for this manuscript. Dr. Wasfy reports a career development award from Harvard Catalyst and the National Institutes of Health (KL2 TRO01100). Dr. Blumenthal reports research funding from the

\section{Compliance with Ethical Standards:}

Conflict of Interest: The authors declare that they do not have a conflict of interest. John S. LaDue Memorial Fellowship at Harvard Medical School. 
Publisher's Note: Springer Nature remains neutral with regard to jurisdictional claims in published maps and institutional affiliations.

\section{REFERENCES}

1. Association of American Medical Colleges. 2013. State Physician Workforce Data Book. https://www.aamc.org/download/362168/data/ 2013statephysicianworkforcedatabook.pdf. Accessed 30 April 2018.

2. Hart LG, Skillman SM, Fordyce M, Thompson M, Hagopian A, Konrad TR. International medical graduate physicians in the United States: changes since 1981. Health Aff (Millwood). 2007;26(4):1159-69. https:// doi.org/10.1377/hlthaff.26.4.1159.

3. Tsugawa Y, Jena AB, Orav EJ, Jha AK. Quality of care delivered by general internists in US hospitals who graduated from foreign versus US medical schools: observational study. BMJ. 2017;356:j273. https://doi. org/10.1136/bmj.j273.

4. Chen LM, Epstein AM, Orav EJ, Filice CE, Samson LW, Joynt Maddox KE. Association of Practice-Level Social and Medical Risk With Performance in the Medicare Physician Value-Based Payment Modifier Program. JAMA. 2017;318(5):453-61. https://doi.org/10.1001/jama.2017.9643.

5. Chen PG, Nunez-Smith M, Bernheim SM, Berg D, Gozu A, Curry LA. Professional experiences of international medical graduates practicing primary care in the United States. J Gen Intern Med. 2010;25(9):947-53. https://doi.org/10.1007/s11606-010-1401-2.

6. Bodenheimer T, Pham HH. Primary care: current problems and proposed solutions. Health Aff (Millwood). 2010;29(5):799-805. https://doi.org/10. $1377 /$ hlthaff.2010.0026. 